\title{
Tecnologias Assistivas Livres para Inclusão de Alunos com Deficiência no Projeto UCA
}

\author{
Ana Grasielle Dionísio Corrêa ${ }^{1}$, Patrícia Araújo de Oliveira ${ }^{1}$, Marilena do Nasci- \\ mento $^{2}$, Irene Karaguilla Ficheman ${ }^{1}$, Gilda Aparecida de Assis ${ }^{1}$, Shirley Takeco \\ Gobara $^{3}$, Elda Gomes Araújo ${ }^{4}$, Roseli de Deus Lopes ${ }^{1}$ \\ ${ }^{1}$ Laboratório de Sistemas Integráveis da Escola Politécnica (LSI-EP) \\ Universidade de São Paulo (USP)
}

Av. Prof. Luciano Gualberto, travessa 3, nº 158, CEP: 05508-970, São Paulo/SP, Brasil

${ }^{2}$ Colmeia Espaço Terapêutico de Medicina Integrada

R. Itararé, 75, Bela Vista, CEP: 01308-030, São Paulo/SP, Brasil

${ }^{3}$ Departamento de Física da Universidade Federal de Mato Grosso do Sul (UFMS) Av. Costa e Silva s/n, Cidade Universitária, CEP: 79070900, Campo Grande/MS, Brasil

${ }^{4}$ Colegiado de Pedagogia da Universidade Federal do Amapá (UNIFAP)

Rodovia JK Zerao, CEP: 68900-000, Macapa/AP, Brasil

\footnotetext{
anagrasi@lsi.usp.br, paaraujo@lsi.usp.br,

marilena@colmeiamedicina.com.br, irene@lsi.usp.br, gildaaa@lsi.usp.br, gobara@dfi.ufms.br, elda@unifap.br, roseli@lsi.usp.br
}

\begin{abstract}
This paper shows the results from a feasibility study of the use of accessibility tools in educational laptops under the UCA project. As results we can get a list of tools chosen by experts, on a pilot test from their experience with disabled children, as well we evaluate the difficulties and satisfactions of children with disabilities who performed activities proposed by the experts on the UCA laptop with the selected tools.
\end{abstract}

Resumo. Este artigo apresenta resultados de um estudo de viabilidade do uso das ferramentas de acessibilidade nos laptops educacionais no âmbito do projeto UCA. Como resultados podemos obter uma lista de ferramentas eleitas por especialistas, em um teste piloto, a partir das suas experiências com crianças com deficiência, assim como avaliar as dificuldades e satisfações de crianças com deficiência que realizaram atividades propostas pelos especialistas no laptop UCA com as ferramentas selecionadas. 


\section{Introdução}

O Projeto Um Computador por Aluno (PROUCA) vem rompendo paradigmas quanto aos saberes relacionados à informática na educação. Desde sua implantação no Brasil, em 2007, o Governo brasileiro tem investido esforços para distribuir laptops educacionais nas escolas e capacitar coordenadores e professores para o trabalho em sala de aula [Gomes 2008]. Essa ação governamental tem proposto mudanças significativas no processo de ensino e aprendizagem por possibilitar a mobilidade dos equipamentos, a conectividade sem fio, a integração dos recursos multimidiáticos tais como vídeos, áudios e câmeras, a imersão dos familiares, entre outras [Rosa et al. 2013]. Desde então, inúmeras pesquisas têm sido realizadas para avaliar o uso e efeitos decorrentes dos laptops educacionais em sala de aula [Struchiner e Giannella, 2012] [Venâncio et al. 2012], a habilidade do professor em incorporá-las ao processo didáticopedagógico [Corrêa et al. 2013] [Nascimento et al. 2011] e os impactos sobre a aprendizagem dos alunos [Bittencourt et al. 2011] [Freire 2009], entre outros aspectos [Quartiero et al. 2012].

As iniciativas de melhorias do trabalho pedagógico nas escolas, seja por meio de políticas de modernização e aporte tecnológico ou pelas ações de capacitação e aperfeiçoamento dos professores não param por ai. Em 2007, o Governo brasileiro instaurou o Decreto 6.094/2007 que estabelece, nas diretrizes do Compromisso Todos pela Educação, a garantia do acesso e permanência no ensino regular e o atendimento às necessidades educacionais especiais dos alunos, fortalecendo seu ingresso nas escolas públicas. Desde então, várias pesquisas sobre acessibilidade sócio digital para pessoas com deficiência podem ser encontradas na literatura [Galvão Filho 2008], sendo predominante o estudo acerca da deficiência visual que, segundo Canal, Almeida e Baranauskas (2012), se deve a motivos como a maior incidência dessa população, cerca de 35,7 milhões de pessoas dos 45,6 milhões de brasileiros com algum tipo de deficiência e suas implicações na computação. De acordo com o último censo demográfico de 2010 realizado pelo Instituto Brasileiro de Geografia e Estatística (IBGE), a deficiência visual, é a incidente (35,7 milhões), seguida da deficiência motora (13,3 milhões), auditiva (9,7 milhões) e mental ou intelectual (2,6 milhões).

Apesar dos inúmeros relatos de experiências positivas com uso dos laptops educacionais nas escolas, estudos envolvendo a acessibilidade e inclusão das crianças com necessidades especiais com uso desses equipamentos e softwares distribuídos com eles ainda são escassos. Canal, Almeida e Baranauskas (2012) realizaram uma pesquisa sobre a dislexia e suas implicações no uso das aplicações do laptop XO. Contudo, o estudo sobre o uso das tecnologias assistivas nos laptops educacionais para apoio à crianças com outros tipos de deficiência precisam também ser contempladas.

Nessa perspectiva, buscamos identificar, analisar e testar um conjunto de tecnologias assistivas nos laptops educacionais UCA, visando favorecer a inclusão de crianças com necessidades especiais nas escolas contempladas com o PROUCA. Para isso, foram necessários os seguintes desdobramentos: levantamento de necessidades das crianças com deficiência nas escolas; levantamento de requisitos de acessibilidade nos laptops educacionais; mapeamento de tecnologias assistivas existentes no mercado brasileiro; seleção de tecnologias assistivas livres para testes funcionais nos laptops UCA; elaboração das atividades pedagógicas para testes com as tecnologias assistivas 
elencadas; testes piloto de avaliação e adequação das ferramentas com especialistas em uso de tecnologias assistivas; testes e avaliações das tecnologias livres nos laptops educacionais com professores e crianças com deficiência.

\section{Metodologia}

Esta seção apresenta a metodologia adotada para a condução desta pesquisa. Inicialmente foi feito um estudo para elencar as atividades pedagógicas a serem desenvolvidas com as crianças com deficiência nas escolas com apoio dos laptops UCA. Em seguida, foi realizado um levantamento de tecnologias assistivas existentes no mercado brasileiro. Após esse levantamento, foram realizados testes de viabilidade técnica das ferramentas encontradas nos laptops UCA. Nessa etapa, cinco ferramentas foram elencadas visando contemplar todas as deficiências. Para coleta de dados, foram criados instrumentos de avaliação das ferramentas e de satisfação dos usuários (professores e alunos). Antes de aplicar a avaliação das ferramentas assistivas nas escolas foi realizado um teste com doze crianças com deficiência motora e uma especialista em Educação Especial.

\subsection{Atividades Pedagógicas}

Para realização deste trabalho foram elaboradas atividades pedagógicas para uso nos laptops por alunos e professores nas escolas. Em Nascimento et al. (2011) são relatadas atividades que foram propostas em um encontro de formação dos professores no Programa UCA. Em um primeiro momento, foi solicitada a exploração dos aplicativos com o objetivo de conhecê-los melhor e avaliar a possibilidade de incorporá-los ao currículo escolar. Em um segundo momento, outra proposta foi apresentada para a apropriação tecnológica do laptop educacional utilizando as próprias ferramentas instaladas na máquina, incluindo aplicativos de escritório (KWord e KPresenter) e jogos educativos (TuxMath, TuxPaint e TuxTyping).

À medida que os professores se familiarizaram com as ferramentas instaladas no laptop, foi possível discutir e elaborar propostas de atividades que poderiam ser desenvolvidas nas salas de aulas juntamente com os alunos. Um instrumento de coleta de dados foi aplicado nos professores com o objetivo de levantar de que forma poderiam conduzir as aulas, integrando o laptop nas atividades pedagógicas pertinentes à rotina da sala de aula. As atividades citadas pelos professores foram: atividades educativas e jogos online para matemática e vídeo nas atividades sobre ciências e saúde; atividades complementares nas áreas de matemática e português onde o aluno tem a oportunidade de aprimorar o seu conhecimento matemático, a oralidade e o seu vocabulário.

Outro levantamento similar foi realizado durante dois Workshops com professores do projeto UCA, que ocorreram na cidade de São Paulo em 2012, onde foram elencados os principais recursos do laptop para as práticas pedagógicas dos professores. As atividades pedagógicas mais citadas pelos professores participantes dos Workshops foram: pesquisa de informações via Internet; escrita de texto; destaque de texto; desenho; cálculo matemático; apresentação e vídeo.

Com base nestes levantamentos, foram elencadas sete atividades (ou tarefas) pedagógicas, apresentadas na Tabela 1 e que foram aplicadas nos testes relatados na Seção 2.5 deste documento. 
Tabela 1. Atividades pedagógicas elencadas

\begin{tabular}{|l|l|}
\hline Tarefa 1 & $\begin{array}{l}\text { Pesquisar o número de medalhas conquistadas pelo Brasil nas Olimpíadas } \\
\text { e Paraolimpíadas de 2012. }\end{array}$ \\
\hline Tarefa 2 & Copiar a frase: “Computador é legal". \\
\hline Tarefa 3 & $\begin{array}{l}\text { Destacar em negrito a palavra "computador" na frase redigida } \\
\text { anteriormente. }\end{array}$ \\
\hline Tarefa 4 & Desenhar uma bola colorida. \\
\hline Tarefa 5 & $\begin{array}{l}\text { Utilizar calculadora para resolver as operações fundamentais de adição } \\
\text { (2+3) e subtração (3-2). }\end{array}$ \\
\hline Tarefa 6 & $\begin{array}{l}\text { Fotografar a si mesmo com a câmera do computador e criar um slide com } \\
\text { a fotografia. }\end{array}$ \\
\hline Tarefa 7 & $\begin{array}{l}\text { Elaborar um vídeo mostrando o ambiente ao redor (sala de aula / } \\
\text { ambiente ) e o professor ou o orientador da atividade. }\end{array}$ \\
\hline
\end{tabular}

\subsection{Seleção das Ferramentas Assistivas}

As ferramentas elencadas para o projeto foram designadas a partir do estudo de viabilidade técnica no laptop UCA, classmate, de baixo custo idealizado para o público apresentando resistência a eventuais quedas [Schneider et al. 2011].

Suas características físicas incluem processador Intel Atom N270, clock de 1.6 gigahertz, capacidade de armazenamento de 4 gigabytes em cartão SD, memória principal de 512 megabytes, tela de cristal líquido de sete polegadas, placa de som com entrada para microfone, microfone embutido e duas caixas acústicas, bateria com autonomia mínima de três horas e peso de $1,5 \mathrm{~kg}$.

$\mathrm{Na}$ busca por um pacote de acessibilidade para o laptop UCA, foram avaliadas três possibilidades de Sistema Operacional (SO): Metasys MeeGo, Lubuntu e Linux Acessível. O Metasys MeeGo foi desenvolvido para dar suporte aos requisitos básicos do processo educativo. Lubuntu, também chamado de light Ubuntu, foi desenvolvido para netbooks, é mais leve, exige menos recursos de hardware e tem uma melhor eficiência energética do que as outras distribuições do Ubuntu. Linux Acessível é uma distribuição Linux direcionada especificamente para pessoas com deficiência visual e tem como principal objetivo o de adicionar, corrigir e configurar os recursos de acessibilidade e usabilidade para pessoas com esta deficiência. A Tabela 2 apresenta a ocupação de memória dos três SOs no laptop UCA.

Tabela 2. Sistema Operacional versus Ocupação de Memória

\begin{tabular}{|c|c|c|c|}
\hline SO & RAM 'livre' & $\begin{array}{c}\text { Armazenamento do } \\
\text { SO }\end{array}$ & $\begin{array}{c}\text { Armazenamento } \\
\text { disponível }\end{array}$ \\
\hline Lubuntu & $400 \mathrm{MB}$ & $3 \mathrm{~GB}$ & $900 \mathrm{MB}$ \\
\hline Linux Acessível & $350 \mathrm{MB}$ & $3 \mathrm{~GB}$ & $900 \mathrm{MB}$ \\
\hline Metasys Meego & $350 \mathrm{MB}$ & $3 \mathrm{~GB}$ & $1 \mathrm{~GB}$ \\
\hline
\end{tabular}


Ao analisar a Tabela 2, observou-se que o SO com melhor desempenho quanto à memória principal é o Lubuntu, pois este se mostrou o mais rápido para ligar e desligar o laptop. Por outro lado, o Metasys Meego proporciona mais espaço de armazenamento livre para futuras instalações.

$\mathrm{Na}$ sequência, foram selecionadas ferramentas de acessibilidade para serem instaladas nos três SOs. São elas: Enable Viacam (eViacam), KdeAccessibility (Kmagnifier, KmouseTool, Kmouth, Kmouth e Jovie), Orca, Mouselupa e Compiz.

Enable Viacam (eViacam) possibilita substituir o mouse por movimentos da cabeça, capturados por uma webcam. KdeAccessibility engloba várias ferramentas de acessibilidade integradas como ampliadores de tela para pessoas com baixa visão (KMagnifier), acionador de botão para pessoas com deficiência física (KmouseTool) e sintetizadores de voz para deficientes visuais (Kmouth e Jovie). Orca proporciona leitura da tela com possibilidade de customização. Mouselupa é uma alternativa de ampliador de tela para uso por pessoas com baixa visão (deficiência visual). Compiz é um gerenciador de janelas que permite a configuração de diversos aspectos das janelas. Nas configurações do Compiz é possível habilitar duas funções adequadas para pessoas com baixa visão: a lupa e o zoom de desktop. O recurso lupa funciona como o MouseLupa e o zoom de desktop permite que o usuário amplie a visão na direção do ponteiro do mouse conforme sua necessidade.

A Tabela 3 apresenta um resumo dos testes de instalação das ferramentas de acessibilidade acima em cada um dos SOs utilizados no laptop UCA.

Tabela 3. Testes de instalação das ferramentas de acessibilidade nos SOs

\begin{tabular}{|c|c|c|c|c|c|}
\hline SO & KdeAcessibility & eViacam & Orca & Compiz & Mouselupa \\
\hline Lubuntu & Instalável & Instalável & Instalável & Instalável & Não funcionou \\
\hline Linux acessível & Instalável & Instalável & Instalado & Instalável & Não funcionou \\
\hline Metasys Meego & Não funcionou & $\begin{array}{c}\text { Não } \\
\text { funcionou }\end{array}$ & $\begin{array}{c}\text { Não } \\
\text { funcionou }\end{array}$ & $\begin{array}{c}\text { Não } \\
\text { funcionou }\end{array}$ & Instalável \\
\hline
\end{tabular}

As opções de SO Lubuntu e Linux acessível se mostram adequadas para o projeto e a escolha do SO Linux acessível foi elencado, pois o pacote de acessibilidade Orca já vem instalado previamente com o SO.

\subsection{Teste de avaliação das ferramentas e instrumentos de Coleta de Dados}

Foi preparado um teste de avaliação das ferramentas escolhidas e foram elaborados instrumentos para coleta de dados para obter opiniões de crianças e de professores: (A) Questionário sobre o perfil do aluno; (B) Questionário para a avaliação de usabilidade; (C) Avaliação da satisfação do usuário/aluno; (D) Questionário de avaliação de aplicabilidade das ferramentas.

O instrumento (A) teve como objetivo coletar dados da criança e sua deficiência, bem como conhecer sua atual experiência com tecnologia da informação. $O$ questionário traçou o perfil da criança, suas possibilidades quanto as habilidades funcionais e a atual vivência com tecnologia da informação. 
O instrumento de avaliação (B) foi elaborado a partir das sete tarefas pedagógicas citadas na Seção 2.1, tendo como objetivos observar a criança enquanto executa as tarefas e verificar se após a orientação recebida ainda precisaria de ajuda ou não, se conclui ou não a tarefa e quanto tempo dispensou para a atividade proposta. São observados os mesmos conceitos para todas as tarefas, e para cada uma delas foi determinado o tempo máximo para sua realização. Foi possível ainda anotar quais as situações que fugiram do comportamento esperado para a execução da tarefa.

$\mathrm{O}$ instrumento de avaliação (C) trata de um questionário aplicado logo após a execução das tarefas pedagógicas e tem por objetivo recolher informações quanto a satisfação do aluno ao realizar as atividades propostas e quanto a utilização das ferramentas assistivas no laptop UCA.

O instrumento (D) foi desenvolvido para ser aplicado com o professor da criança com deficiência, o professor responsável pelo do laboratório de informática da escola ou outro técnico que transite entre o aluno e as atividades pedagógicas com a tecnologia da informação dentro da estrutura escolar. Esta pessoa tem como pressuposto o desafio dentro do ambiente escolar de entender e propor soluções para a inclusão da criança com necessidades especiais. A opinião da pessoa designada para este papel quanto as questões de aplicabilidade das ferramentas assistivas propostas é de grande relevância.

Foram conduzidas entrevistas com cinco especialistas em atendimento de crianças com deficiência com o objetivo de testar, avaliar e ajustar os instrumentos de coleta de dados. As experiências pessoais destes especialistas, no exercício do seu trabalho junto a criança especial, permitiram identificar os termos mais adequados para a linguagem da criança, o formato e conteúdo das perguntas avaliativas, e os comportamentos esperados para as atividades propostas.

\subsection{Teste Piloto com Especialistas}

Primeiramente foi realizada a avaliação da aplicabilidade das ferramentas eleitas com cinco profissionais (especialistas ou professores) sendo que todos tinham vivencia com o uso de computadores e utilizaram ou conheciam algum recurso de acessibilidade. As ferramentas eleitas foram: leitor de tela, eViacam e teclado virtual.

A partir do uso das ferramentas escolhidas e aplicação dos questionários de avaliação de usabilidade (B) e de aplicabilidade das ferramentas (D) com os especialistas e professores, foram ajustados os questionários de satisfação (C) que levantariam a opinião da criança quanto a proposta das ferramentas escolhidas, ou seja, a melhor adaptação para a necessidade da criança dentro da possibilidade real no ambiente escolar.

\subsection{Testes com Crianças com Deficiência}

Nesta fase do projeto foram realizados testes em duas escolas distintas, com doze crianças que foram acompanhadas por especialistas. Entre as crianças que participaram dos testes cerca de 35\% tinham algum comprometimento visual, 93\% comprometimento físico e $71 \%$ precisaram de acompanhamento especial.

Todas as crianças possuem computador, sendo que $17 \%$ possuem computador somente na escola, 50\% somente em casa e 33\% em ambos lugares. $93 \%$ já haviam utilizado computador anteriormente, porém nenhuma utilizou o laptop UCA 
anteriormente. Apenas $42 \%$ possuem acesso à Internet.

Devido a essas peculiaridades separamos os resultados em dois momentos: primeiramente apresentamos (Tabela 4) o número de crianças que precisaram de ajuda para realizar tarefas e o número de crianças que concluíram as tarefas. A partir disso, juntamos as opiniões das crianças que realizaram os testes segundo o grau de diversão, facilidade e utilidade das atividades realizadas.

A primeira tarefa "pesquisar o número de medalhas conquistadas pelo Brasil nas Olimpíadas e Paraolimpíadas de 2012" foi omitida nos resultados, pois não pôde ser realizada por nenhuma das doze crianças por falta de conexão à Internet nos locais onde foram aplicados os testes.

Tabela 4. Número de crianças em relação a necessidade de ajuda e conclusão das tarefas

\begin{tabular}{|c|c|c|c|c|c|c|}
\hline & $\begin{array}{c}\text { Tarefa } \\
2\end{array}$ & $\begin{array}{c}\text { Tarefa } \\
3\end{array}$ & $\begin{array}{c}\text { Tarefa } \\
4\end{array}$ & Tarefa 5 & $\begin{array}{c}\text { Tarefa } \\
6\end{array}$ & $\begin{array}{c}\text { Tarefa } \\
7\end{array}$ \\
\hline $\begin{array}{c}\text { Número de crianças que } \\
\text { precisaram de ajuda para realizar } \\
\text { as tarefas }\end{array}$ & 4 & 6 & 7 & 5 & 5 & 6 \\
\hline $\begin{array}{c}\text { Número de crianças que } \\
\text { concluíram as tarefa }\end{array}$ & 11 & 9 & 10 & 9 & 9 & 9 \\
\hline
\end{tabular}

Observou-se que as principais dificuldades enfrentadas foram por dificuldades de compreensão das tarefas pelas crianças, necessidades especiais que impediram de concluir ou realizar a tarefa (como movimentações involuntárias e atividades com os pés, por exemplo) e limitações no hardware.

Quanto ao grau de satisfação das crianças, os resultados foram promissores, apesar das dificuldades citadas anteriormente. A maioria, que conseguiu concluir as atividades propostas, as classificaram como "legal" ou "muito legal" segundo critério de diversão, "fácil" ou "muito fácil" no critério de facilidade e "útil" ou "muito útil" no critério de utilidade, como mostram os gráficos (a), (b) e (c), respectivamente, na Figura 1. Foram considerados na porcentagem de satisfação apenas aqueles que conseguiram concluir a tarefa.

Os especialistas que conduziram os testes sugeriram que as letras do teclado tenham um maior contraste visual e aderência, e pontuaram o uso de adesivos como uma possível solução. 


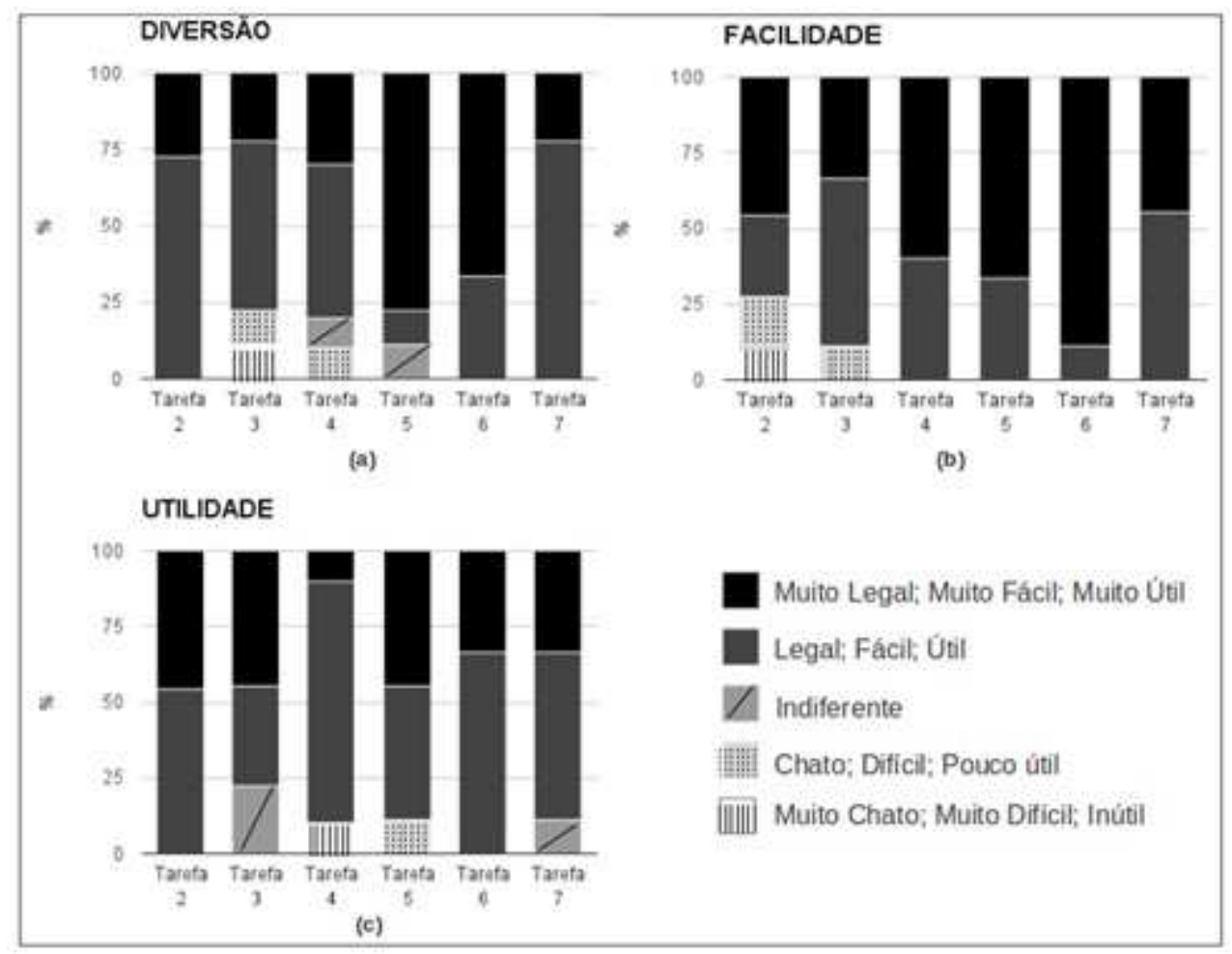

Figura 1. Satisfação na realização das tarefas

\section{Conclusões}

A proposta inicial do Projeto UCA - Um Computador por Aluno é de disponibilizar um laptop de baixo custo para cada aluno da unidade escolar. No entanto, observou-se que crianças com deficiência não foram atendidas por este projeto, pois lhe faltavam ferramentas de interação com os laptops. Com isso, a criança com deficiência vem sendo excluída das atividades pedagógicas conduzidas com os laptops UCA.

Com o intuito de fornecer subsídios para a inclusão de crianças com deficiência nas escolas UCA, este projeto teve por objetivo levantar e testar ferramentas acessíveis livres para os laptops UCA. Foram selecionadas três ferramentas e foram conduzidos testes das mesmas com 12 crianças com deficiência.

Com os resultados parciais alcançados até o momento, é possível afirmar que as ferramentas escolhidas ajudarão algumas crianças com deficiência.

Como trabalhos futuros, pretendemos relatar os resultados dos testes que estão sendo conduzidos com as crianças com deficiência nas escolas, acompanhadas por professores nos Estados de São Paulo (SP), Mato Grosso do Sul (MS) e Amapá (AP), onde serão verificadas a aplicabilidade das ferramentas no ambiente escolar. Esperamos conseguir resultados promissores para que seja possível contemplar e incluir todas as crianças que frequentam as escolas. 


\section{Referências}

Bittencourt, J.; Kist, S.O.; Tatizana, D.; Schäfer, P.B. e Fagundes, L.C. (2012), "Ambientes virtuais de aprendizagem na modalidade 1:1: um estudo de caso sobre o AMADIS na Fase I do Programa UCA", Revista Novas Tecnologias na Educação (RENOTE), v.9, n.2, 2011. Canal, M.C.; Almeida, L.D.; Baranauskas, M.C.C Uma avaliação de acessibilidade no laptop educacional da OLPC na perspectiva de pessoas com dislexia, Anais do $23^{\circ}$ Simpósio Brasileiro de Informática na Educação (SBIE 2012), Rio de Janeiro.

Canal, M.C.; Almeida, L.D. e Baranauskas, M.C.C (2012), "Uma avaliação de acessibilidade no laptop educacional da OLPC na perspectiva de pessoas com dislexia", Anais do $23^{\circ}$ Simpósio Brasileiro de Informática na Educação (SBIE 2012), Rio de Janeiro.

Corrêa, A.G.D; Venâncio, V.; Ficheman, I. K. e Lopes, R.D. (2013), "Relato de Experiências de Oficinas Pedagógicas para a Formação de Professores do Projeto UCA", In: Congresso Brasileiro de Recursos Digitais na Educação, 2013, São Paulo. II Congresso Brasileiro de Recursos Digitais na Educação, pp. 1-10.

Freire, K.X.(2009), "UCA: Um Computador por Aluno e os Impactos Sociais e Pedagógicos”, Anais do IX Congresso Nacional de Educação (EDUCERE), pp. 5889-5899.

Galvão Filho, T. e Damasceno, L. (2008) "Tecnologia Assistiva em Ambiente Computacional: Recursos para a Autonomia e Inclusão Sócio-Digital da Pessoa com Deficiência", In: Instituto de Tecnologia Social - ITS. (Org.), Tecnologia Assistiva nas Escolas: Recursos Básicos de Acessibilidade Sócio-Digital para Pessoas com Deficiência, 1ed., São Paulo: ITS, v. 1, pp. 25-38.

Gomes, A. (2008) "Um computador por aluno: a experiência brasileira", Relator P. H. Lustosa, Equipe técnica C. A. Lopes, A. P. de Queiroz Filho e A. L. Camelo, Brasília: Câmara dos Deputados, Coordenação de Publicações, p. 195 (Série avaliação de políticas públicas, 1).

Quartiero, E.M.; Fantin, M.; Bonilla, M.H. e Pretto, N.L. (2012), “Gestão e Práticas Pedagógicas no Âmbito do Programa UCA: desafios e estratégias à consolidação de uma política pública para a educação básica”, Projeto Um Computador por Aluno: pesquisas e perspectivas. Sampaio, F.F; Elia, M.F. (Org), Rio de Janeiro: NCE/UFRJ, pp. 68-78.

Nascimento, K.A.S.; Filho, J.A.; Salgueiro, G.L. e Sales, S.B. (2011), "Um olhar sobre a formação docente do programa UCA em uma escola municipal de Fortaleza", Anais do XVII Simpósio Brasileiro de Informática na Educação (SBIE), Workshop de Informática na Escola (WIE), pp. 1448-1547.

Rosa, V.; Coutinho, C.; Coelho da Silva, J.L.; Souza, C. e Rosa, S. (2013), "Programa Um Computador por Aluno no Brasil", In: M. J. Gomes, A. Osório, A. Ramos, B. Silva \& L. Valente (Orgs.), Challenges 2013: Aprender a qualquer hora e em qualquer lugar, learning anytime anywhere, Atas da VIII Conferência Internacional de TIC na Educação, pp. 61-71. Braga: Centro de Competência TIC do Instituto de 
Educação da Universidade do Minho.

Schneider, F. C.; Santarosa, L. M. C. e Conforto, D. (2011) "Cidade Um Computador por Aluno - UCA Total A identificação de situações inclusivas na totalidade". In: Simpósio Brasileiro de Informática na Educação, 2011, Aracajú. Anais do SBIE, 2011.

Struchiner, M. e Gianella, T.R. (2012), “Análise do Processo de Integração de Tecnologias de Informação e Comunicação em Atividades no Ensino Fundamental no Contexto do Programa Um Computador por Aluno (PROUCA)", Projeto Um Computador por Aluno: pesquisas e perspectivas. Sampaio, F.F; Elia, M.F. (Org), Rio de Janeiro: NCE/UFRJ, pp. 32-43.

Venâncio, V.; Corrêa, A.G.D.; Telle, E.O.; Corrêa, C.M.; Garcia, B.V.R.; Ribeiro, C.C.; Ficheman, I.K. e Lopes, R.D. (2012), "Integração dos Laptops Educacionais ao Cotidiano Escolar no UCA São Paulo: facilidades e dificuldades", In: I Congresso Brasileiro de Recursos Digitais na Educação, São Paulo. 